\title{
Objects earlier than precursors of UC HII regions: Inflow-signpost for a common way of star formation
}

\author{
Y. $\mathbf{W u}^{1}$, M. Zhu ${ }^{2}$, D. $\mathrm{Xu}^{1}, \mathrm{Y} \cdot \mathrm{Wei}^{1}$ and L. $\mathrm{Zhu}^{1}$ \\ ${ }^{1}$ Department of Astronomy, Peking University, Beijing 100871, China, \\ email: yfwu@bac.pku.edu.cn \\ ${ }^{2}$ Joint Astronomy Centre/National Research Council of Canada, Herzberg Institute of \\ Astrophysics, 5071 West Saanich Road, Victoria, BC V9E 2E7, Canada
}

\begin{abstract}
Searching for objects in the earliest phases of star formation, e.g sources at the beginning of a gravitational collapse, are essential to our understanding of massive star formation. Today a number of precursors of ultra compact HII regions (PUCHs) have been found. Embedded in dense gas and dust, these PUCHs have a high bolometric luminosity but little or no $6 \mathrm{~cm}$ radio continuum emission (Molinari, et al. 2000; Beuther, et al. 2002). Evidence for Collapse was found in ultra compact (UC) HII regions and 12 water maser sources (Zhang, et al. 1998; Wu \& Evans II 2003). This paper presents the identification of massive cores with no detectable infrared and radio sources. These kinds of cores usually have strong sub-mm emission. A special case is the SCUBA core JCMT 18354-0649S which has both infall and outflow motions as indicated by the profiles of high excitation molecular lines. This core is at a stage earlier than PUCHs. Blue profiles are also found in UC HII region, which indicates that material is still infalling in this phase. Our observations suggest that infall exists in different evolutionary stages for high mass star formation, similar to the low mass cases.
\end{abstract}

Keywords. ISM: cloud - ISM: kinematics and dynamics - stars: formation

\section{Introduction}

Characterizing high mass young stellar objects (YSOs) in their early phases is the key to understanding massive star formation. Infall motion, if detected, would present a direct evidence to support the idea that massive stars form via a process similar to that of low mass stars. However, the nature for high mass star formation is very difficult to clarify because of the complexity of such regions and their large distance from us. Besides, their rapid evolution and ever changing conditions make it difficult to probe the processes inside the protostellar condensations amoung highly obscured surroundings. Many surveys were carried out to search for high mass YSOs in recent years (Wood \& Churchwell 1989; Bronfman, et al. 1996; Plume, et al. 1997; Zinchenko, et al. 1997; Mueller, et al. 2002; Shirley, et al. 2003; Molinari, et al. 1996; Molinari, et al. 2002; Zhang, et al. 2001; Sridharan, et al. 2002; Beuther, et al. 2002). A number of precursors of ultra compact HII regions (PUCHs) were found (Molinari, et al. 2002; Beuther, et al. 2002; Churchwell 2002 and reference therein). The IRAS colors of PUCHs are similar to that of UC HII regions (Wood \& Churchwell 1989) or very young stellar objects (Richards et al. 1987). Their IRAS flux densities are as high as $\mathrm{f}_{60}>100 \mathrm{Jy}$ or $\mathrm{f}_{100}>500 \mathrm{Jy}$ (Molinari, et al. 1996; Sridharan, et al. 2002). They are also characterized by strong $\mathrm{mm}$ or sub-mm emission. Their spectral energy distribution is rather flat and molecular outflow detection rate is as high as $84 \%$ to $90 \%$ (Sridharan, et al. 2002; Zhang, et al. 2001). The important 

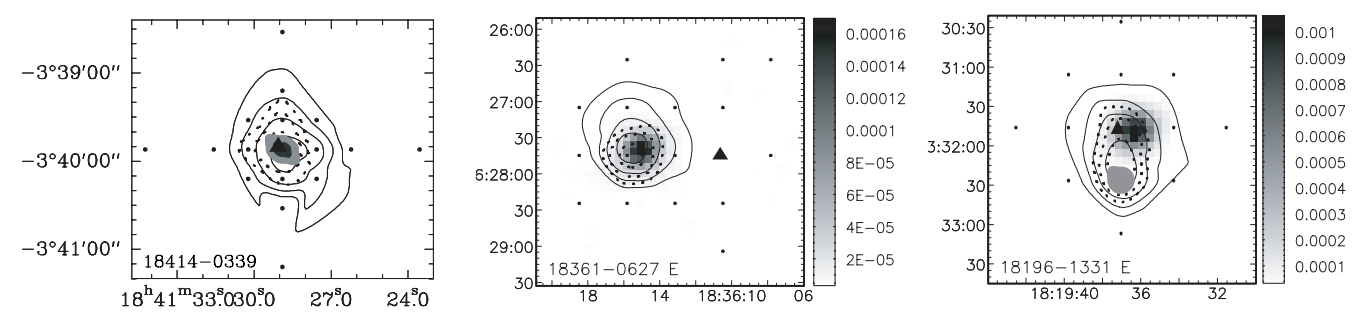

Figure 1. The left panel: Ammonia core IRAS 18414-0339 with emission peak (contours) coincidence with IRAS source (triangle). The middle pannel: Ammonia core IRAS 18361-0627 with emission peak overlapped with MSX E band image (grey scales). The right panel: Ammonia core IRAS 18196-1331 with emission peak deviated from any IRAS (triangle) and MSX E band source (grey scales). The MSX flux density is in $\mathrm{Wm}^{-2} \mathrm{sr}^{-1}$ (Wu et al. 2005a).

indications for these objects being massive and young are the high luminosity $\mathrm{L}_{b}>10^{3} \mathrm{~L}_{\odot}$ and the lack of $6 \mathrm{~cm}$ radio continuum emission.

Infall motions were detected in 12 high mass star formation regions by Wu \& Evans II (2003). Inverse P-Cygni profile was found in W51 several years ago (Zhang, et al. 1998). Recently, $\mathrm{NH}_{3}(3,3)$ line and radio continuum studies have revealed that the molecular accretion flow becomes ionized accretion flow when passing through the ionization front (Sollins et al. 2005).

This paper presents the preliminary results in our search for objects earlier than PUCHs and a search for inflow motions in different phases of high mass star formation.

\section{A search for objects earlier than PUCHs}

\subsection{Massive gas cores not associated with infrared sources}

Massive molecular cores that contain no infrared sources are potential objects for star formation at a very early phase. In an ammonia survey, with a sample similar to that of Molinari, et al. (1996) and Sridharan, et al. (2002), 5 sources with peak ammonia emission deviated from IRAS sources were detected (Wu, et al. 2005a). The angular distance between the amonia peak and IRAS peak are more than one telescope beam (40"). Further identification with Midcourse Space Experiment (MSX) shows that one of them is overlapped with an infrared source, and the other 4 contains 5 cores that are separated from the peak emission at MSX A and E bands. These five cores have a mass of $780 \mathrm{M}_{\odot}$ on average and the FWHM of line $(1,1)$ is $1.80 \mathrm{~km} \mathrm{~s}^{-1}$, much larger than those of low mass cores (Myers, et al. 1983). The detection of such cores are common in molecular line maps. In the detected sources of Zinchenko, et al. (1997), half are deviated from IRAS sources. In the survey of Harju, et al. (1993) in the Orion and Cepheus Clouds, a number of cores defined as minor cores are not associated with IRAS sources. Part of their major cores are also deviated from IRAS sources. Jijina, et al. (1999) listed all the ammonia cores without SIMBAD sources. We also detected several such cores with CO isotopes despite their low excitation density and depletion (Wei, et al. 2004). Fig. 1 presents the examples for three types of cores: cores with ammonia peak overlapped with IRAS, MSX sources or deviated from any IRAS and MSX sources. Cores deviated from infrared sources either harbor near infrared sources without $6 \mathrm{~cm}$ emission or are located beside an HII region.

\subsection{Sub-millimeter emission}

Sub-millimeter emission is important for studying dense cores which are too cold to be detectable at mid infrared. Here we present recent SCUBA observations of the $\mathrm{NH}_{3}$ core 

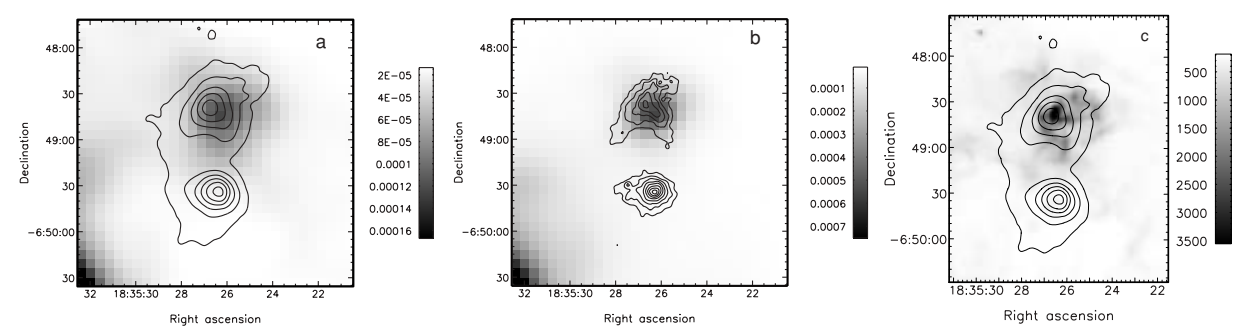

Figure 2. a) SCUBA $850 \mu \mathrm{m}$ contours on MSX A band image $(6.8-10.8 \mu \mathrm{m})$. Contour levels at $850 \mu \mathrm{m}$ are from $0.71(10 \sigma)$ to 4.25 by $0.71 \mathrm{Jy} /$ beam. b) SCUBA $450 \mu \mathrm{m}$ contours on MSX E band image $(18.2-25.1 \mu \mathrm{m})$. Contour levels are from $2.9(5 \sigma)$ to 17.7 by $2.1 \mathrm{Jy} /$ beam. The MSX flux density is in $\mathrm{Wm}^{-2} \mathrm{sr}^{-1}$. c) SCUBA $850 \mu \mathrm{m}$ contours on IRAC $8 \mu \mathrm{m}$ band image. The $8 \mu \mathrm{m}$ flux density scale is in MJy $s r^{-1}$. Contour levels are the same as in a). (Wu et al. 2005b).

18354-0649 (Wu, et al. 2005a). This core is located near IRAS 18355-0650. But these two objects are not at the same distance because the $\mathrm{V}_{L S R}$ for the IRAS source and $\mathrm{NH}_{3}$ core are $65 \mathrm{~km} \mathrm{~s}^{-1}$ and $95 \mathrm{~km} \mathrm{~s}^{-1}$ respectively (Lester, et al. 1985). However, the $\mathrm{NH}_{3}$ core is associated with an HII region G25.4 NW with $\mathrm{V}_{L S R}$ of $95 \mathrm{~km} \mathrm{~s}^{-1}$. The distance is $5.7 \mathrm{kpc}$. SCUBA detected strong continuum emission at both 850 and $450 \mu \mathrm{m}(\mathrm{Wu}$, et al. 2005b). Fig. 2 shows the overlay of a) $850 \mu \mathrm{m}$ contours on MSX A band; b) $450 \mu \mathrm{m}$ contours on E band; c) $850 \mu \mathrm{m}$ contours on IRAC $8 \mu \mathrm{m}$ image. The diameter of the compact dust core is 10" according to the measured FWHM in the 450 mum map (after deconvolved with the 8 " beam). The dust temperature is $14.4 \mathrm{~K}$ (if $\beta=2$ ) and $25.2 \mathrm{~K}$ (if $\beta=1.5)$. This core is named as JCMT 18354-0649S.

Using $\mathrm{M}=\mathrm{S}_{\nu} \mathrm{D}^{2} / \mathrm{k}_{\nu} \mathrm{B}_{\nu}\left(\mathrm{T}_{d}\right)$ and assuming $\mathrm{T}_{d}=20 \mathrm{~K}$, the core mass was estimated as $820 \mathrm{M}_{\odot}$ according to the $450 \mu \mathrm{m}$ flux density. The average gas density is $1.1 \times 10^{6} \mathrm{~cm}^{-3}$ in the central region with a radius of 5". These parameters suggest that the core is massive and dense. They may be similar to the massive cores detected by Garay, et al. (2004). Zhu \& Wu (2005) also identified a number of such cores from the previous mm and sub-mm surveys. The optical extinction Av of the core JCMT 18354-0649S estimated with the peak flux density at $850 \mu \mathrm{m}$ is 630 (wu, et al. 2005b).

\subsection{Investigation with high density molecular tracers}

The following high excitation molecular lines were observed toward the core JMCT 183540649S using the JCMT in May 2004, including $\mathrm{HCN}(3-2), \mathrm{HCO}^{+}(3-2), \mathrm{H}^{13} \mathrm{CO}^{+}(3-2)$, $\mathrm{CO}(3-2)$ and $\mathrm{C}^{17} \mathrm{O}(2-1)$. Fig. 3a and $3 \mathrm{~b}$ present the grid of $\mathrm{HCN}(3-2)$ and $\mathrm{HCO}^{+}(3-2)$ respectively, which are $3 \times 3$ with 10 " spacing. In total there were four pairs of optically thick and thin lines (Fig. 3c), all with the signature of collapse in the central region: the ratio of $\mathrm{T}_{k}$ (Blue) $/ \mathrm{T}_{k}$ (Red) $>1$ for both $\mathrm{HCN}(3-2)$ and $\mathrm{HCO}^{+}(3-2) ; \delta \mathrm{v}=\left(\mathrm{V}_{\text {thick }}{ }^{-}\right.$ $\left.\mathrm{V}_{\text {thin }}\right) / \Delta \mathrm{V}_{\text {thin }}<-0.25$ (Mardones, et al. 1997) for all pairs. These results show that there is infall motion in this core. The radii of the infall region was estimated to be 8".

An analytic model for collapse (provided by Myers, et al. (1996)) was tested on JCMT 18354-0648S (Fig. 3d and 3e). This model assumes that the cloud consists of two uniform paralleled components without rotation. The resulting parameters $\left(\mathrm{T}_{k}, \sigma, \tau_{0}, \mathrm{~V}_{i n}\right)$ from the model fitting are much larger than the corresponding values of low mass sources, consistent with the high mass nature of the core. The kinematic mass infall rate is 

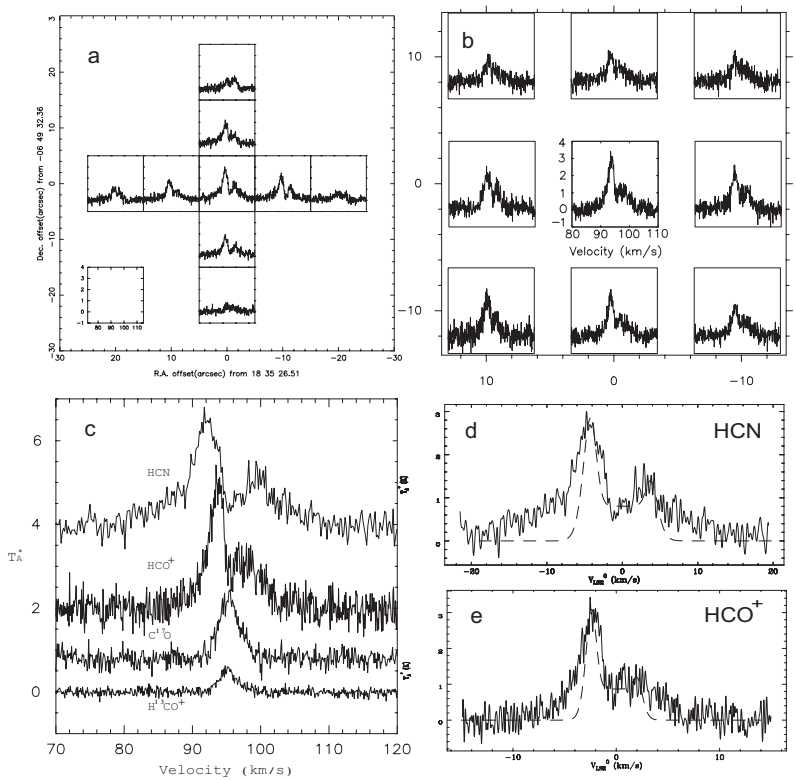

Figure 3. Spectral lines of the SCUBA core JCMT 18354-0649S. a) HCN (3-2) grid with 10" spacing. $\mathrm{X}$ and $\mathrm{Y}$ axis are the R.A. and DEC. offset in arcsec, respectively. b) $\mathrm{HCO}^{+}(3-2)$ grid with 10" spacing. $\mathrm{X}$ and $\mathrm{Y}$ axis are the same as a). c) $\mathrm{HCN}(3-2), \mathrm{HCO}^{+}(3-2), \mathrm{C}^{17} \mathrm{O}(2-1)$ and $\mathrm{H}^{13} \mathrm{CO}^{+}(3-2)$ lines at the central position. d) and e) $\mathrm{HCN}$ and $\mathrm{HCO}^{+}(3-2)$ spectra and model fitting (dashed line) in zero velocity frame (indicated as $V_{\mathrm{LSR}}^{0}$ ). (Wu et al. 2005b).

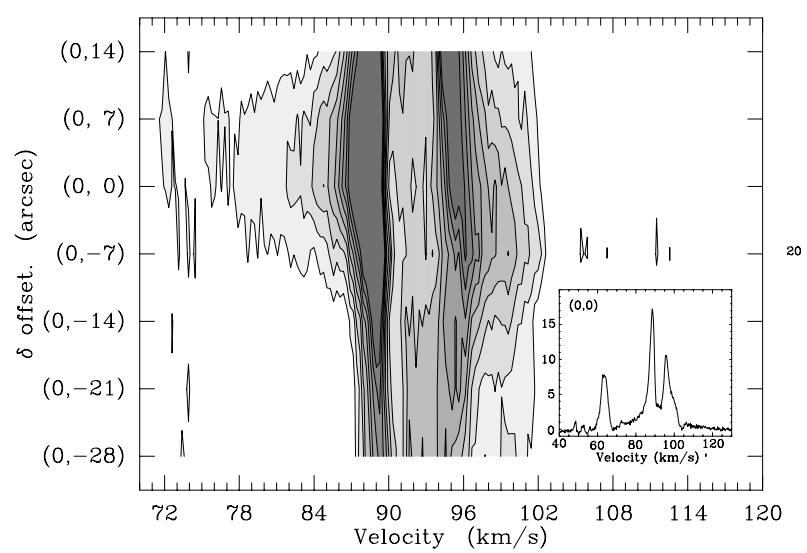

Figure 4. Position-velocity diagram of $\mathrm{CO}(3-2)$ spectra along $\Delta R . A .=0$. Contour levels are from $3 \sigma$ to $27 \sigma$ by $3 \sigma(1 \sigma=0.345 \mathrm{~K}$. Spectrum at the lower-right corner is at $(0,0)$ position R.A. $(1950)=18^{\mathrm{h}} 35^{\mathrm{m}} 26.5^{\mathrm{s}}$, DEC. $(1950)=-06^{\circ} 49^{\prime} 32^{\prime \prime}$.

$3.4 \times 10^{-3} \mathrm{M}_{\odot} / \mathrm{yr}$ which is calculated according to the formula in Myers, et al. (1996) $\mathrm{dM} / \mathrm{dt}=4 \pi \mathrm{R}_{\text {in }} \mathrm{mnV}_{\text {in }}$. The gravitational rate is $1.4 \times 10^{-3} \mathrm{M}_{\odot} / \mathrm{yr}$. The ratio of these two values is within a factor of 2 for low mass sources (Myers et al. 1996), but a bit higher in our case. CO (3-2) line was observed in a cross pattern with a grid step of 10". The total line width reaches $38 \mathrm{kms}^{-1}$. A position-velocity diagram in Fig. 4 shows that there is a bipolar outflow in this region. The existence of infall and outflow motions, combining with the unusual values of the physical parameters, indicates that there is a high mass star being formed inside the core. The fact that no MSX and near-infrared emission 

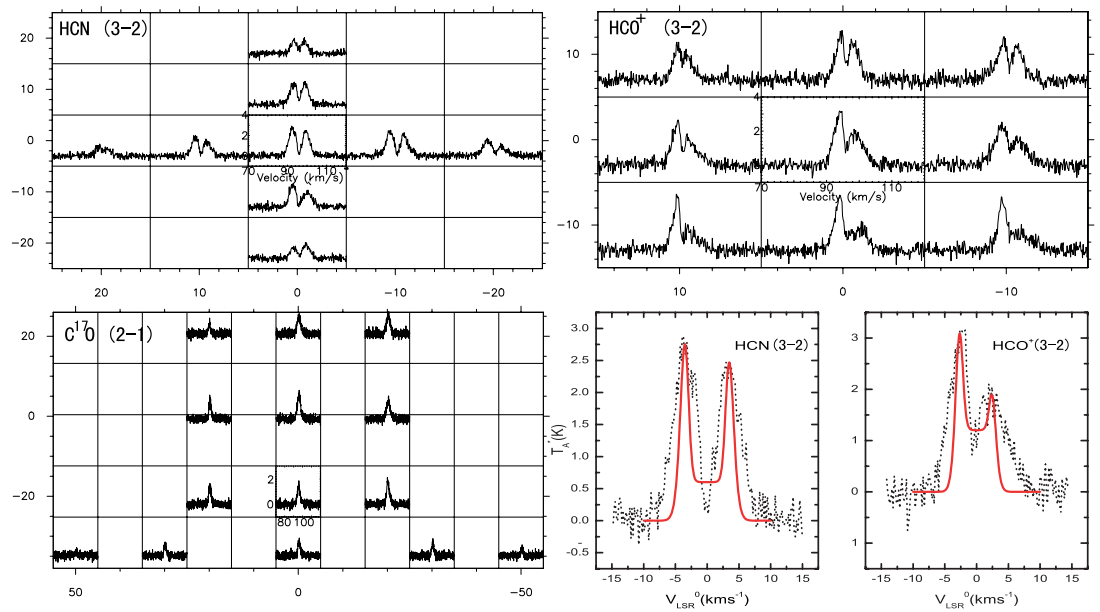

Figure 5. Spectral lines of the northern SCUBA core JCMT 18354-0649N. a) HCN (3-2) grid with 10". $\mathrm{X}$ and $\mathrm{Y}$ axis are the R.A. and DEC. offset in arcsec, respectively. b) $\mathrm{HCO}^{+}(3-2)$ grid with $10 " . \mathrm{X}$ and $\mathrm{Y}$ axis are the same as a). c) $\mathrm{HCN}(3-2), \mathrm{HCO}^{+}(3-2), \mathrm{C}^{17} \mathrm{O}(2-1)$ and $\mathrm{H}^{13} \mathrm{CO}^{+}(3-2)$ lines at the central position. d) and e) $\mathrm{HCN}$ and $\mathrm{HCO}^{+}(3-2)$ specatra and model fitting (dashed line) in zero velocity frame (indicated as $\mathrm{V}_{L S R}^{0}$ ).

are detected is likely due to the extremely high extinction of the core. High resolution observations are necessary to provide a detailed picture and to examine the nature of this core.

\section{Inflow motions in different evolutionary phases}

In Fig. 2, there is a core overlapped with G25.4NW located north to JCMT 183540649S. G25.4NW is a well studied HII region. Infrared observations reveal that its dust temperature is $59 \mathrm{~K}$ (Lester, et al. 1985). The bolometric luminosity is $\mathrm{L}_{b}=5.6 \times 10^{5} \mathrm{~L}_{\odot}$ $(5.7 \mathrm{kpc})$. From Fig. 2, one can see that its dust emission is more extended than that of the southern one, and of a higher $\mathrm{T}_{d}$. Spectral line observations were also followed up at this source. Fig. 5 presents the spectra of $\mathrm{HCN}(3-2), \mathrm{HCO}^{+}(3-2)$ and $\mathrm{C}^{17} \mathrm{O}(2-1)$. There were at least two pairs of optically thick and thin lines. The ratio of $\mathrm{T}_{k}$ (Blue) $/ \mathrm{T}_{k}(\mathrm{Red})$ for $\mathrm{HCN}(3-2)$ and $\mathrm{HCO}^{+}(3-2)$ are 1.2 and 1.9 respectively; and $\delta \mathrm{v}$ from $\mathrm{HCN}(3-2)$, $\mathrm{HCO}^{+}(3-2)$ and $\mathrm{C}^{17} \mathrm{O}(2-1)$ are -0.52 and -0.48 respectively. All these satisfy the criteria for a blue profile (Mardones et al. 1997). Model analysis was also performed. The HCN and $\mathrm{HCO}^{+}(3-2)$ spectra and the model fitting (solid line) in zero velocity frame (noted with $\left.\mathrm{V}^{0}{ }_{L S R}\right)$ ) is also shown in Fig. 5 (lower-right panel). The infall velocities derived from fitting the $\mathrm{HCN}(3-2)$ and $\mathrm{HCO}^{+}(3-2)$ data are 0.03 and $0.16 \mathrm{kms}^{-1}$ respectively, less than those in JCMT 18354-0649S. These results indicate that the core containing HII region G25.4NW is also collapsing. Obviously, this core is in an evolutionary stage different from that of JCMT 18354-0649S. For low mass sources, collapse features in different evolutionary phases were well known. In Table 1 we present the examples of collapse candidates in different evolutionary phases for both low and high mass sources. Although the evolutionary phases of low and high mass sources can not be compared with each other directly, the examples show that infall motions are presented in high mass star formation from a phase earlier than PUCHs to the phase of UC HII regions, just as it exists in different evolutionary stages of the low mass star forming process. 
Table 1. collapse candidates in different stages of star formation

\begin{tabular}{lccc}
\hline Source & \multicolumn{3}{c}{ Evolutionary phases } \\
\hline Low mass $^{1,2}$ & Pre-protostellar & Class 0 & Class I \\
Examples & L1544 & VLA 16293, B335 & L1251B, WL22 \\
\hline High mass & Earlier than PUCHs & PUCHs & HII \\
Examples & JCMT 18354-0649S & W3NE $^{3}$ ? & G25.4NW \\
\hline
\end{tabular}

Note: 1. Shirley et al. 2000; 2. Andre et al. 2000; 3. Wei et al. 2004.

\section{Acknowledgements}

We are grateful to the JCMT staff for all their assistance with the observations. This project was supported by the Grant 10133020, 10128306, 10203003 of NSFC and G1999075405 of NKBRSF.

\section{References}

Andre, P., Ward-Thompson, D., Barsony, M. 2000 in: V. Mannings, A.P. Boss, S.S. Russell (ed.), Univ. of Arizona press Tucson 2000 Protostars and planets IV p. 59

Beuther, H., Schilke, P., Menten, K.M., Motte, F., Sridharan, T.K., Wyrowski, F. 2002, ApJ, 566,965

Bronfman, L., Nyman, L.A., May, J. 1996, A\&AS, 115, 81

Churchwell, E. 2002, ARA\&A, 40, 27

Clark, F.O., Laureijs, R.J., Prusti, T. 2002, ApJ, 731, 602

Garay, G., Faundez, S., Mardones, D., Bronfman, L., Chini, R., Nyman, L. 2004, ApJ 610, 313

Harju, J., Walmsley, C.M., Wouterloot, J.G.A. 1993, A\&AS 98, 57

Jijina, J., Myers, P.C., Adams, F.C. 1999, ApJS 125, 161

Lester, D.F., Dinerstein, H.L., Werner, M.W., Harvey, P.M., Evans II, N.J., Brown, R.L. 1985, ApJ, 296, 565

Mardones, D., Myers, P.C., Tafalla, M., Wilner, D.J., Bachiller, R., Garay, G. 1997, ApJ, 489, 719

Molinari, S., Brand, J., Cesaroni, R., Palla, F. 1996, A\&A, 308, 573

Molinari, S., Brand, J., Cesaroni, R., Palla, F. 2000, A\&A, 355, 617

Molinari, S., Testi, L. Rodriguez, L.F., Zhang, Q. 2002, ApJ, 570, 758

Mueller, K.E., Shirley, Y.L., Evans II, N.J., Jacobson, H.R. 2002, ApJS, 143, 469

Myers, P.C., Mardones, D., Tafalla, M., Williams, J.P. Wilner, D.J. 1996, ApJ, (letter) 465, L133

Myers, P.C., Linke, R. A., Benson, P. J. 1983, ApJ, 264, 517

Plume, R., Jaffe, D.T., Evans II, N.J., Martin-Pintado, J., Gomez-Gonzalez, J. 1997, ApJ, 476, 730

Richards, P.J., Little, L.T., Toriseva, M., Heaton, B.D. 1987, MNRAS, 228, 43

Shirley Y.L., Evans II, N.J., Rawlings, J.M.C., Gregersen, E.M. 2000, ApJS, 131, 249

Shirley Y.L., Evans II, N.J., Young, K.E., Knez, C., Jaffe, D.T. 2003, ApJS, 149, 375

Sollins, P.K., Zhang, Q., Keto, E., Ho, P.T.P. 2005, ApJ, (letter) 624, L49

Sridharan, T.K., Beuther, H., Schilke, P., Menten, K.M., Wyrowski, F. 2002, ApJ, 566, 931

Wei, Y., Wu, Y., Wang, Y., Xu, D., Ju, B. 2004, JKAS in press

Wu, J., Evans II, N.J. 2003, ApJ, (letter) 592, L79

Wu, Y., Zhang, Q., Yu, W., Miller, M., Mao, R., Sun, K., Wang, Y. 2005, submitted to A\&A

Wu, Y., Zhu, M., Wei, Y., Xu, D., Zhang, Q., Fiege, J.D. 2005, ApJL (accepted)

Zhang, Q., Ho, P.T.P., Ohashi, N. 1998, ApJ, 494, 636

Zhang, Q., Hunter, T.R., Brand, J., Sridharan, T.K., Molinari, S., Kramer, M.A., Cesaroni, R. 2001, ApJ, (Letters) 552, L167

Zhu, L., Wu, Y. 2005, In preparation

Zinchenko, I., Henning, Th., Schreyer, K. 1997, A\&GAS, 124, 385 